\title{
Stabilization of continuous fluidized bed spray granulation - a Lyapunov approach
}

\author{
Stefan Palis ${ }^{1}$, Achim Kienle ${ }^{1,2}$ \\ 1 Otto-von-Guericke-Universität, \\ Universitätsplatz 2, D-39106 Magdeburg, Germany \\ (corresponding author e-mail: stefan.palis@ovgu.de) \\ 2 Max-Planck-Institut für Dynamik komplexer technischer Systeme \\ Sandtorstrasse 1, D-39106 Magdeburg, Germany
}

\begin{abstract}
This article deals with the stabilization of continuous fluidized bed spray granulation with external product classification. Using Lyapunov stability theory a control law is derived guaranteeing exponential convergence of the error particle size distribution in a norm associated to the second moment. In contrast to other approaches popular for particle process control no model reduction is used.
\end{abstract}

Keywords: Chemical Process; Lyapunov Methods

\section{INTRODUCTION}

Granulation is one of the most important manufacturing processes in food and pharmaceutical industries. It is used to produce granules from liquid products, e.g. solutions or suspensions.

In Fig. 1 a typical process flow sheet is shown. It consists of a granulation chamber, two sieves to remove under and oversized particles from the product and a mill. It has been shown [2] that this configuration of continuous fluidized bed spray granulation with external product classification and material recycles shows unstable behavior for certain ranges of the operating parameters. The instability results in nonlinear oscillations of the particle size distribution, which gives normally undesired time variance of product properties. Possible solutions are a redesign, i.e. avoiding parameter combinations connected to a region of instability, or a stabilization via feedback.

The second approach should be preferred as it gives the possibility to operate the process in the full range of operating parameters. In addition to that feedback control allows a direct adjustment of the desired product properties and rejects unforeseen disturbances.

The main difficulties regarding the control design arise from the complicated plant model, as the dynamics are described by a first order nonlinear partial integrodifferential equation, the population balance equation, with sinks and sources in the domain. For continuous crystallization comparable dynamical behavior has been observed. Here the main approaches for control are mostly based on model reduction, i.e. through discretization [4], approximation of the particle size distribution with a series expansion [5], or linearization [6].

In this contribution a completely different approach based on Lyapunov stability theory for distributed parameter systems as in [7], [8] is proposed not using any model reduction.

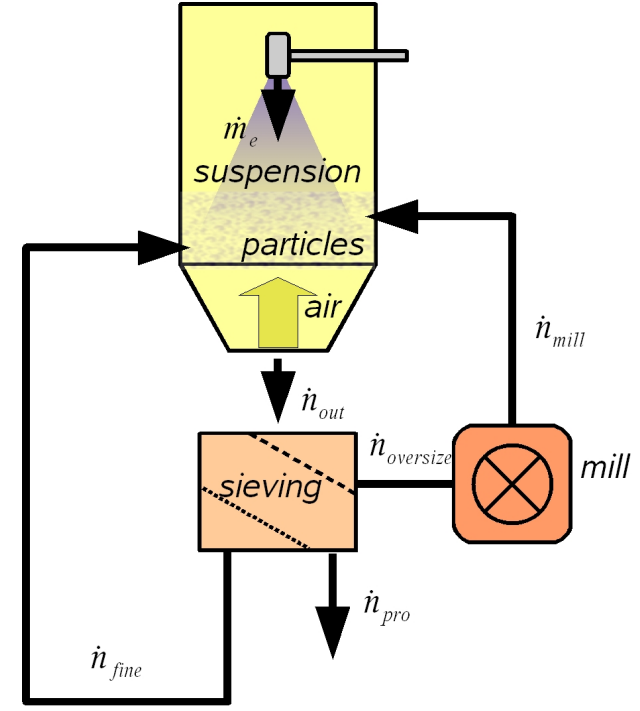

Fig. 1. Scheme of the granulation process

\section{MODEL OF A CONTINUOUS FLUIDIZED BED SPRAY GRANULATION}

The main assumptions for the model of a continuous fluidized bed spray granulation are:

- nonporous, spherical particles,

- no agglomeration or breakage,

- ideal mixing.

The granulator consists of a granulation chamber, where the particle population is fluidized through an air stream and coated by injecting a suspension $\dot{m}_{e}$. The associated particle growth has been described in [1].

$$
G=\frac{2 \dot{m}_{e}}{\varrho A}=\frac{2 \dot{m}_{e}}{\varrho \pi \mu_{2}}
$$


In the continuous configuration of the fluidized bed spray granulation particles are continuously removed in order to achieve a constant bed mass, which correlates to a constant third moment of the particle number distribution $\mu_{3}=\int_{0}^{\infty} L^{3} n d L$. The particle flux being removed from the granulator is:

$$
\dot{n}_{\text {out }}=K n \text {. }
$$

where $K$ is the drain which follows from the assumption of a constant bed mass and which is derived later. The removed particles are then sieved in two sieves and separated into three classes:

(1) Fine particles, which are directly fed back to the granulator,

$$
\dot{n}_{\text {fines }}=\left(1-T_{2}\right)\left(1-T_{1}\right) \dot{n}_{\text {out }}
$$

(2) Product particles, which are removed from the whole process,

$$
\dot{n}_{\text {prod }}=T_{2}\left(1-T_{1}\right) \dot{n}_{\text {out }}
$$

(3) Oversized particles, which are grinded in a mill and fed back to the ganulator.

$$
\dot{n}_{\text {oversize }}=T_{1} \dot{n}_{\text {out }}
$$

Here the separation functions $T_{1}$ and $T_{2}$ (Fig. 2) for the two screens are as follows:

$$
T_{1 / 2}=\frac{\int_{0}^{L} e^{-\frac{\left(L-\mu_{1 / 2}\right)^{2}}{2 \sigma_{1 / 2}^{2}}} d L}{\int_{0}^{\infty} e^{-\frac{\left(L-\mu_{1 / 2}\right)^{2}}{2 \sigma_{1 / 2}^{2}}} d L} .
$$

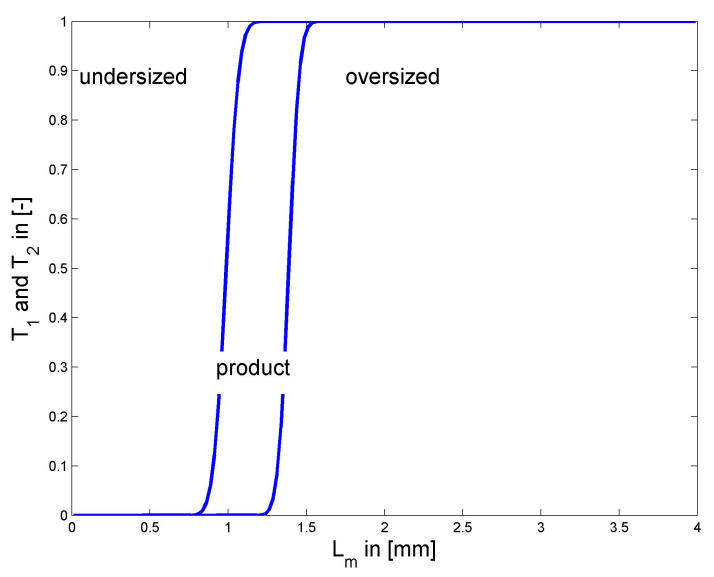

Fig. 2. Separation functions $T_{1}$ and $T_{2}$

To describe the process, a population balance model for the particle size distribution has been derived in [2]. In this contribution a simplified modell is used neglecting external seeding, attrition and overspray. Nevertheless, open-loop simulation gives comparable results.

In the model fine particles are directly fed back to the granulator, which results in a cancellation of the associated sink and source. Hence the simplified population balance equation consists of the following particle fluxes:

- $\dot{n}_{\text {prod }}$ particle flux due to product removal,

- $\dot{n}_{\text {oversize }}$ particle flux due to oversize removal,

- $\dot{n}_{\text {mill }}$ particle flux due to particles fed back from mill,

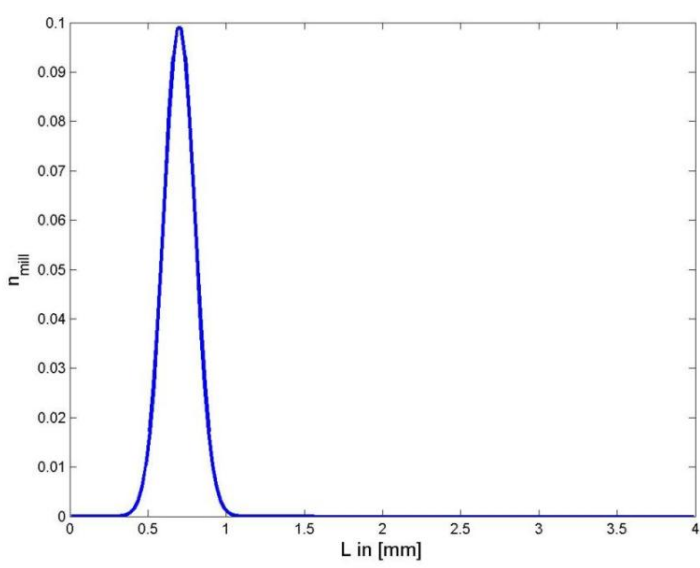

Fig. 3. Distribution of milled particles $n_{m i l l}$ for $\mu_{M}=0.7$ and particle growth associated with the size independent growth rate $G$.

$$
\frac{\partial n}{\partial t}=-G \frac{\partial n}{\partial L}-\dot{n}_{\text {prod }}-\dot{n}_{\text {oversize }}+\dot{n}_{\text {mill }}
$$

The particle distribution fed back from the mill is assumed to be a normal distribution, where the mean diameter $\mu_{M}$ represents the mill grade. In the following the mill grade $\mu_{M}$ will be used as the control input. For physical reasons $\mu_{M}$ should not exceed a lower $(0.5 \mathrm{~mm})$ and upper limit $(0.9 \mathrm{~mm})$.

$$
\dot{n}_{\text {mill }}=6 \frac{e^{-\frac{\left(L-\mu_{M}\right)^{2}}{2 \sigma_{M}^{2}}}}{\sqrt{2 \pi} \pi \varrho \sigma_{M}} \int_{0}^{\infty} L^{3} \dot{n}_{\text {oversize }} d L
$$

Assuming ideal mass control the drain $K$ is calculated such that the first time derivative of $\mu_{3}$ becomes zero implying a constant bed mass.

$$
\begin{aligned}
\dot{\mu}_{3} & =\int_{0}^{\infty} L^{3} \frac{\partial n}{\partial t} d L=0 \\
& =\int_{0}^{\infty} L^{3}\left[-G \frac{\partial n}{\partial L}-\dot{n}_{\text {oversize }}-\dot{n}_{\text {prod }}+\dot{n}_{\text {mill }}\right] d
\end{aligned}
$$

Because the mill is assumed to be mass conserving the third moments of the oversize flux and mill flux are equal resulting in:

$$
\begin{aligned}
0 & =\int_{0}^{\infty} L^{3}\left[-G \frac{\partial n}{\partial L}-\dot{n}_{\text {prod }}\right] d L \\
& =3 G \int_{0}^{\infty} L^{2} n d L-K \int_{0}^{\infty} L^{3} T_{2}\left(1-T_{1}\right) n d L .
\end{aligned}
$$

Here the fact that the particle size distribution vanishs at the boundary $\left(n(0, t)=\lim _{L \rightarrow \infty} n(L, t)=0\right)$ has been used for integration by parts. Solving equation (12) for the drain $K$ gives:

$$
K=\frac{3 G \int_{0}^{\infty} L^{2} n d L}{\int_{0}^{\infty} L^{3} T_{2}\left(1-T_{1}\right) n d L} .
$$

For an extended model, i.e. with external seeding, attrition and overspray, an extensive bifurcation study has 
been undertaken in [3]. It has been shown, that for sufficiently small mill grades $\mu_{M}$ self-sustained oscillations occur. This circumstance can be explained by the fact, that for fine grinding the population surface excessively increases resulting in a very small growth rate. Thus the particle flux from oversize fraction vanishes, which gives a slowly growing population of small particles. When this population reaches the oversize fraction the whole process repeats. Analogous patterns of behavior are observed for the simplified model considered in this contribution.

Therefore for a specific mill grade, i.e. at the bifurcation point, the stationary particle size distribution loses its stability and an additional limit cycle occurs [3]. In the following a control law is designed in order to stabilize the stationary particle size distribution in the whole range of $\mu_{M}$

\section{CONTROLLER DESIGN}

In order to design a control law the error $e$ is defined as:

$$
e=\int_{0}^{\infty} L^{2}\left(n_{d}-n\right) d L
$$

It should be mentioned, that the error is an integral quantity of the difference between desired particle size distribution $n_{d}$ and the process particle size distribution. The choice is motivated by the fact, that the particle growth depends on the surface area of the particle size distribution, which is strongly connected to its second moment. To derive a controller the following candidate Lyapunov function is introduced:

$$
V=\frac{1}{2} e^{2} .
$$

The time derivative of $V$ along the system trajectories (7) is:

$$
\begin{aligned}
\dot{V}= & e \dot{e}=-e \int_{0}^{\infty} L^{2} \frac{\partial n}{\partial t} d L \\
= & -e\left[\int_{0}^{\infty} 2 L G n-L^{2}\left(T_{1} K n+T_{2}\left(1-T_{1}\right) K n\right) d L\right. \\
& \left.+\int_{0}^{\infty} L^{3} T_{1} K n d L \int_{0}^{\infty} L^{2} n_{\text {mill }} d L\right]
\end{aligned}
$$

Here $n_{\text {mill }}$ is the shape of the particle size distribution generated by the mill depending on the mill grade $\mu_{M}$. As the second moment of $n_{\text {mill }}$ cannot be directly solved for the mill grade $\mu_{M}$ the characteristic curve (Fig. 3) has been inverted pointwise (Fig. 3). In the following the second moment of $n_{\text {mill }}$ will therefore be used as a virtual control $u_{\text {virt }}$.

$$
u_{v i r t}=\int_{0}^{\infty} L^{2} n_{m i l l}\left(\mu_{M}\right) d L
$$

Using (17) the negative definiteness of the time derivative of the candidate Lyapunov function $V$ can be guaranteed choosing the following virtual control law.

$$
\begin{aligned}
u_{v i r t}= & \frac{1}{\int_{0}^{\infty} L^{3} T_{1} K n d L}\left[c e-\int_{0}^{\infty} 2 L G n\right. \\
& \left.-L^{2}\left(T_{1} K n+T_{2}\left(1-T_{1}\right) K n\right) d L\right]
\end{aligned}
$$

In addition to stability the control law (19) even guarantees exponential convergence of $V$.

$$
\dot{V}=-c e^{2}=-2 c V
$$
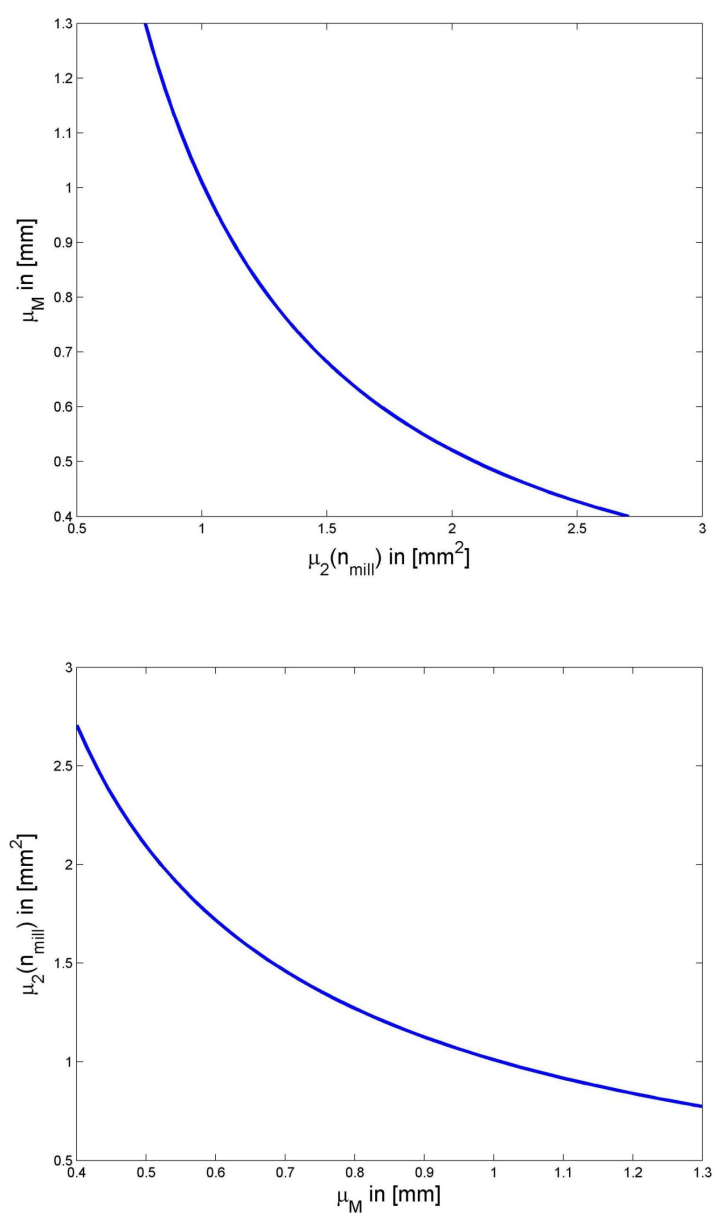

The resulting control scheme (Fig. 3) therefore consists of the control law (19) using the virtual control $u_{\text {virt }}$ and an inversion of the characteristic curve from $\int_{0}^{\infty} L^{2} n_{m i l l}\left(\mu_{M}\right) d L$ to $\mu_{M}$.

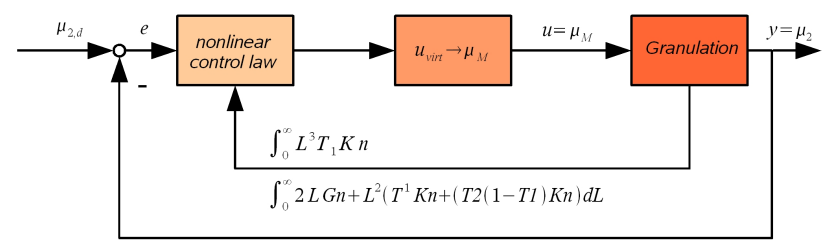

\section{SIMULATION}

For the numerical simulation the population balance has been spatially discretized using a finite volume method with an upwind scheme. The spatial mesh contains 160 equidistant points in the domain of $0<L<4 \mathrm{~mm}$.

\begin{tabular}{|l|l|}
\hline \multicolumn{2}{|c|}{ Parameters } \\
\hline$\rho$ & $1.610^{-3} \frac{\mathrm{g}}{\mathrm{mm}^{3}}$ \\
$\dot{m}_{e}$ & $\frac{100}{3.6} \frac{\mathrm{g}}{\mathrm{s}}$ \\
$m_{\text {init }}$ & $100 \mathrm{~kg}$ \\
$\mu_{1}$ & $1.4 \mathrm{~mm}$ \\
$\sigma_{1}$ & $0.055 \mathrm{~mm}$ \\
$\mu_{2}$ & $1 \mathrm{~mm}$ \\
$\sigma_{2}$ & $0.065 \mathrm{~mm}$ \\
$\mu_{M}$ & $0.9 \mathrm{~mm}$ \\
$\sigma_{M}$ & $0.1 \mathrm{~mm}$ \\
\hline
\end{tabular}


To test the proposed control law the process is started in the region of stability (for example $\mu_{M}=0.9$ ). Then the particle size distribution is shifted into the region of instability by continuously increasing the desired second moment $\mu_{2, d}$ or decreasing the mill grade $\mu_{M}$ in openloop operation. As can be seen in Fig. 4 the process becomes unstable in open-loop operation showing increasing oscillations, which would finally reach the associated limit cycle [3]. Applying the proposed control law the process is stabilized and oscillations occurring during the shifting are damped. The particle size distribution and all its moments $\mu_{0}, \mu_{1}, \mu_{2}$ (Fig. 5) are stabilized with reasonable control effort.

For an implementation on the real plant either the appropriate moments from equation (19) have to be measured or calculated from the measured particle size distribution. A particle size distribution measurement can be achieved by for example by spatial filter velocimetry measurement, focused beam reflectance measurement or in-process video microscopy. Depending on the measurement principle an transformation from chord length distribution to particle size distribution might by necessary, which is uncritical as in fluidized bed spray granulation typically spherical particles are produced.

\section{CONCLUSION AND FUTURE WORK}

A controller for continuous fluidized bed spray granulation with external product classification has been derived. It has been shown to give exponential convergence in a norm associated to the second moment. Simulation results indicate that although having convergence in a norm the whole particle size distribution and its moments $\mu_{0}$ and $\mu_{1}$ are stabilized. So far the proposed control law assures convergence only in an idealized setting, i.e. no parameter and no model uncertainty. Thus further investigations will be made on robustification and adaption.

\section{REFERENCES}

[1] L. Mörl \& M. Mittelstrass \& J. Sachse, Zum Kugelwachstum bei der Wirbelschichttrocknung von Lösungen oder Suspensionen. Chem. Techn. 29, 1977, Heft 10, pp. 540-542.

[2] S. Heinrich \& M. Peglow \& M. Ihlow \& M. Henneberg \& L. Mörl, Analysis of the start-up process in continuous fluidized bed spray granulation by population balnce modelling, Chem. Eng. Sci. 57, 2002, pp. 4369-4390.

[3] R. Radichkov, T. Müller, A. Kienle, S. Heinrich, M. Peglow, L. Mörl, A numerical bifurcation analysis of continuous fluidized bed spray granulation with external product classification, Chem. Eng. and Proc., vol. 45, issue 10, Oct. 2006, pp. 826-837.

[4] R. Eek, Control and dynamic modelling of industrial suspension crystallizers, Ph.D. thesis, TU Delft, 1995.

[5] P. Christofides \& N. El-Farra \& M. Li \& P. Mhaskar, Modelbased control of particulate processes, Chem. Eng. Sci. 63, 2008, pp. 1156-1172.

[6] U. Vollmer \& J. Raisch, Population balance modelling and $H_{\infty^{-}}$ controller design for a crystallization process, Chem. Eng. Sci., vol. 57, issue 20, Oct. 2002, pp. 4401-4414.

[7] T. Sirasetdinov, Stability of systems with distributed parameters [in Russian], 1987.

[8] A. Martynjuk \& R. Gutovski, Integral inequalities and stability of motion [in Russian], Naukowa Dumka, 1979. 

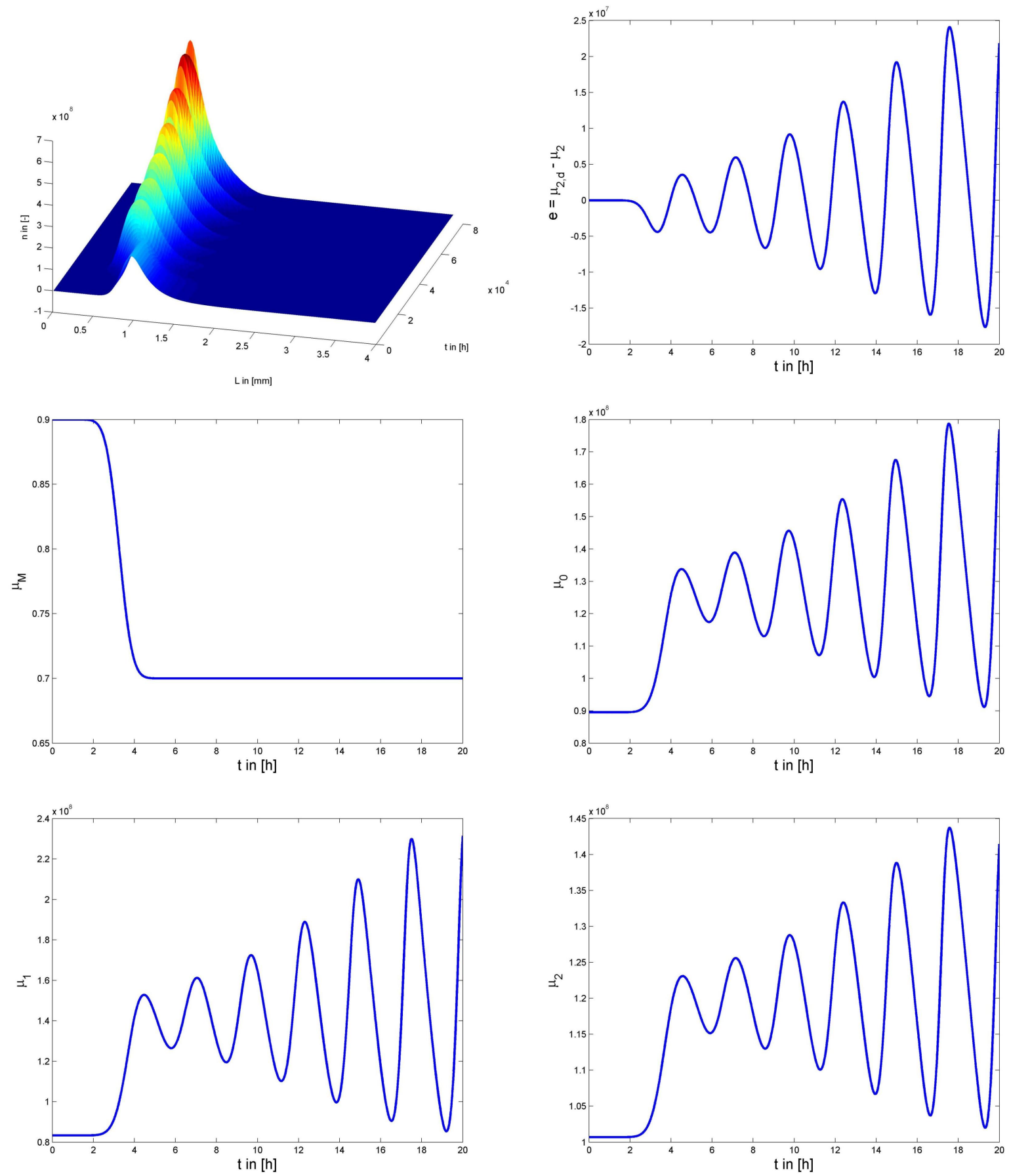

Fig. 4. Open loop system response 

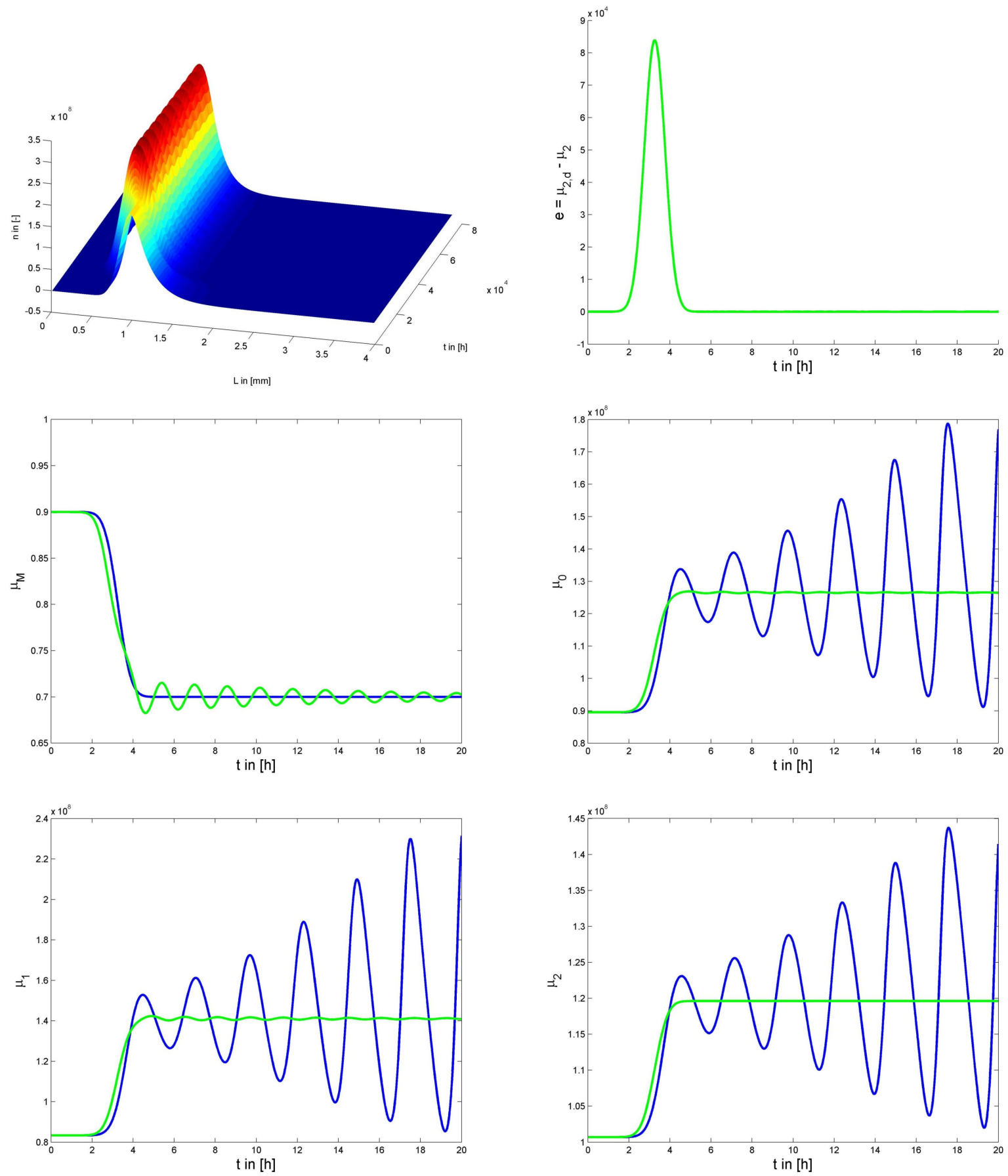

Fig. 5. Open loop (blue) and closed loop (green) system response 